\title{
GALACTIC WINDS
}

\author{
Heinrich J. Völk ${ }^{1}$, D. Breitschwerdt ${ }^{1}$, J.F. McKenzie ${ }^{2}$ \\ ${ }^{1}$ Max-Planck-Institut für Kernphysik, Heidelberg \\ ${ }^{2}$ Max-Planck-Institut für Aeronomie, Lindau
}

\section{Introduction}

In the sequel we will be discussing that - apart from all other halo flows - it is highly probable that our Galaxy has a systematic mass loss; most other galaxies should behave in a similar way. This mass loss should occur in the form of a supersonic galactic wind provided the intergalactic pressure is low enough. We will investigate this possibility mainly for our Galaxy. We shall argue [1] that the energetic particle component of the interstellar medium, the cosmic rays (CRs), is basically responsible for driving a wind for typical gas temperatures below $10^{6} \mathrm{~K}$. Thus for asymptotic wind velocities of the order of the escape velocity, the mass flux is essentially determined by the observable CR energy loss from the Galaxy. Galaxies with very hot gas on the other hand hardly need the CR component and drive a wind almost exclusively through their thermal pressure.

\section{Is the halo on the average static or convective?}

Presumably the gas flows in the halo above the disk (defined by the cool neutral hydrogen gas layer with a half-thickness of about $100 \mathrm{pc}$ ) are of a three-dimensional nature [2]. Therefore an upward flow at one point above the disk does not imply that an upward flow must occur elsewhere as well. On the contrary, even with locally nonzero mass flows the halo can be static on average with zero average mass velocity. From a gas dynamic point of view such a halo is entirely compatible with a Galactic fountain picture [3,4], where upwelling parcels of gas reach finite heights above the plane to fall back afterwards. However it is not clear whether the CRs can escape through the gas to extragalactic space withough dragging part of the gas with them. At least this appears as an extreme assumption. As another possibility the halo could on average be convective. This could either be due to a mass loss, say in the form of a wind, or due to mass accretion. Mass accretion could occur in the form of infall of extragalactic clouds as advocated a long time ago by Oort [5] and suggested by the $21 \mathrm{~cm}$ observations of Mirabel and Morras [6] and van Woerden et al. [7]. Again, due to the three-dimensional nature of the halo flows, either wind or infall, or both simultaneously might occur, together with a system of fountains. In all cases the CRs will escape to "infinity". We consider a (stochastic) combination of wind outflow and cloud accretion as most likely. 


\section{Cosmic Ray Component in the Galaxy}

The CRs constitute a High Energy Component of the interstellar medium (ISM) which appears to be quasi-steady with a pressure in the disk $p_{c d} \simeq 3 \cdot 10^{-13} \mathrm{dyn}_{\mathrm{cm}}^{-2} \lesssim p_{g d} \simeq$ $6 \cdot 10^{-13} \mathrm{dyn} \mathrm{cm}^{-2}$, where $p_{g d}$ is the pressure of the thermal disk gas. The outward CR energy flux density at the disk boundary, based on the spallation grammage of $\mathrm{GeV}$ particles, is $F_{c d}=3.6 \cdot 10^{40} \mathrm{erg} \mathrm{sec}^{-1} \cdot A_{\text {gal }, d}^{-1}$, where $A_{\text {gal,d }}$ is the surface area of the galactic disk (e.g. [8]). Actually, because the grammage decreases with increasing particle energy, $F_{c d}$ should be multiplied by a factor of order 3 . The total energy loss $\simeq 10^{41} \mathrm{erg} / \mathrm{sec}$ due to CRs then corresponds to about 10 percent of the energy produced by supernovae in the Galaxy (assuming one supernova of $10^{51}$ erg per 30 years). There is no radiative cooling of the CR component. Upon its drift through the gas it excites MHD waves, basically Alfvén waves. These waves tend to propagate outwards into the halo. CR particles scatter on the associated magnetic fluctuations and thus transfer outward momentum to the gas. The basic alternative is whether there occurs an outflow together with the gas or whether the particles just diffuse outwards leaving the gas behind.

\section{Halo dynamies}

In analogy to the solar corona we consider the galactic magnetic field configuration as a mixture of open and closed field lines. Open field lines should be due to the production of hot gas and CRs by OB stars and supernova remnants in the upper disk, or due to the Parker instability of a stratified medium [9]. The CRs diffuse in the frame of the outward propagating Alfvén waves towards $|z|=\infty$, perpendicular to the plane of the Galaxy (Fig. 1). This combination of Alfvénic drift and diffusion of the CRs through the gas [10] constitutes a form of "heat conduction". Ionisation of the thermal gas should be effectively maintained by upper disk OB stars, halo stars and globular cluster stars from "within", and quasars from "outside". At heights $|z| \gtrsim 1 \mathrm{kpc}$ above other (hydrodynamic) wave sources the CR pressure gradient should lead to a very strong resonant generation of waves in the fully ionized halo gas. As a result, the coupling of the thermal plasma and the CRs is strong (e.g. [11]). To lowest approximation we then can neglect CR diffusion keeping only their drift with the waves through the plasma. Together with the wave pressure gradient this implies a sizeable outward force density on the thermal gas. The neglect of CR diffusion in the halo (but not in the disk up to $|z| \simeq 1 \mathrm{kpc}$ !) is consistent with an energy dependent escape of the CRs, as it is inferred from observations.

Thus we consider an adiabatic flow of a 3-component system consisting of thermal gas, waves and CRs (cf. [12]) at heights $|z| \gtrsim$ few kpc above the galactic midplane. Neglecting forces due to the average magnetic field $\underline{B}$, we adopt a one-dimensional flux tube model with "radially" diverging flux tubes at distances exceeding the radius of the galactic disk. Such a model neglects centrifugal effects with their obvious tendency to strengthen any wind flow. 


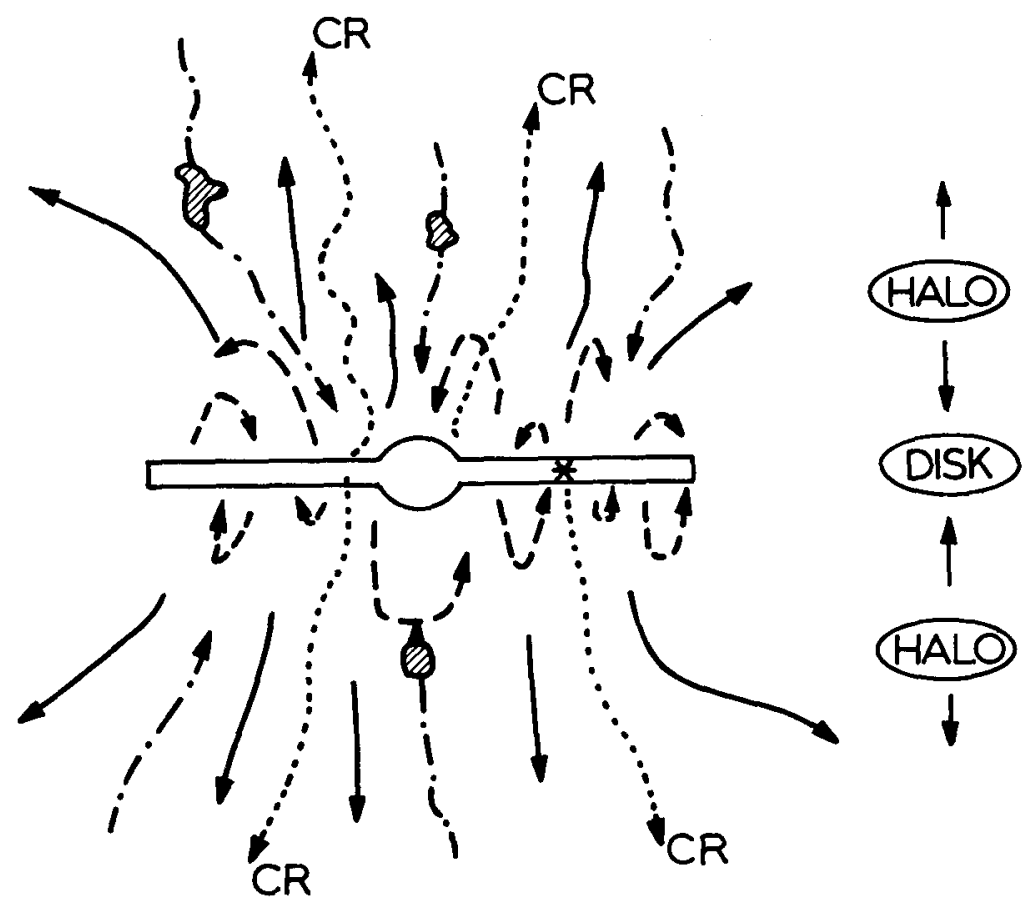

Figure 1: Cartoon of the various possible halo flows. The dashed lines correspond to a fountain flow. The dash-dot lines indicate extragalactic clouds (hatched) falling onto the Galaxy disk, indicated schematically. A galactic mass loss flow is given by the solid lines, whereas the escaping CRs correspond to the dotted flow lines.

\section{Sample solutions}

The hydrodynamical theory is described in a number of papers $[13,14,15]$. Here we will confine ourselves to a discussion some sample results. Taking the inner boundary at $\left|z_{0}\right|=1 \mathrm{kpc}$ (reference level), with a density $n_{0}=10^{-3} \mathrm{H}$-atoms $\mathrm{cm}^{-3}$ and a gas temperature $T_{0}=10^{8} \mathrm{~K}$, a small CR pressure $p_{c o}=10^{-13}$ dyn $\mathrm{cm}^{-2}$, and $B_{0}=1 \mu \mathrm{G}$, we take a gravitational potential due to a massive dark halo $\left(8 \cdot 10^{11} M_{\odot}\right)$ apart from a disk $\left(2.5 \cdot 10^{11} M_{\odot}\right)$ and a bulge $\left(2 \cdot 10^{10} M_{\odot}\right)$ contribution [16]. The wave pressure $p_{w o}=$ $10^{-2} \cdot B_{0}^{2} / 8 \pi$ is taken quite small at the reference level to obtain a conservative estimate.

For a magnetic flux tube at a distance $R_{0}=10 \mathrm{kpc}$ from the galactic center the resulting flow exhibits a critical point at a height of $\left|z_{c}\right|=34 \mathrm{kpc}$. This implies that the intergalactic pressure is sufficiently small. The flow speed $u_{0}$ at the reference level is about $10 \mathrm{~km} \mathrm{sec}-1$, reaching an asymptotic terminal velocity $u_{f}=310 \mathrm{~km} \mathrm{sec}-1$ at $|z| \gg\left|z_{c}\right|$. The CR and wave pressures dominate the gas pressure for $|z| \geq 10 \mathrm{kpc}$, demonstrating that the wind is basically driven by the CRs which have a much softer equation of state than the gas (Fig. 2). 


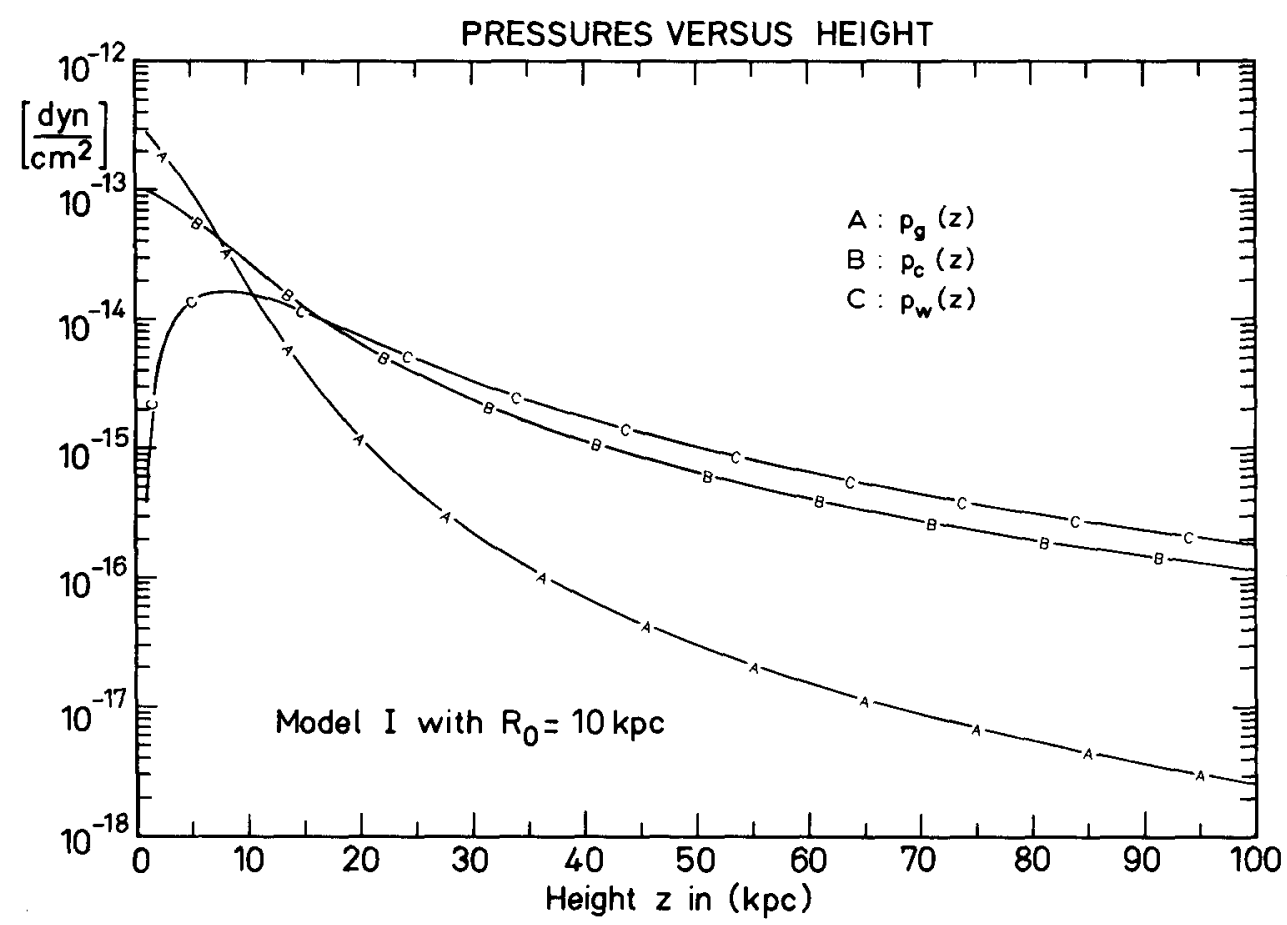

Figure 2: Results of an outflow calculation along a flux tube $|z|>\left|z_{0}\right|$, starting at the radial distance $R_{0}=10 \mathrm{kpc}$ in the disk and a reference level $\left|z_{0}\right|=1 \mathrm{kpc}$ above it. The three pressures $p_{g}, p_{c}$, and $p_{w}$, given by the symbols $\mathrm{A}, \mathrm{B}$, and $\mathrm{C}$ on the curves correspond to the pressures of the thermal plasma, the CRs, and the scattering waves.

Keeping, in a simple-minded approach, the boundary conditions at $\left|z_{0}\right|$, while varying $R_{0}$, shows a monotonic increase (decrease) of $n_{0} u_{0} u_{f}$ with decreasing $R_{0}, u_{f}$ being about twice the escape speed from the point $\left|z_{0}\right|, R_{0}$. The resulting galactic mass loss rate is $\dot{M}_{g a l} \simeq 0.3 M_{\odot} / y r$ for the conservative CR energy loss rate $F_{c d} \cdot A_{g a l, d}=3.6 \cdot 10^{40} \mathrm{erg}$ $\sec ^{-1}$ quoted above.

Assuming $\dot{M}_{g a l} \simeq$ const. for a galactic age of the order of $10^{10} \mathrm{yr}$ implies that over this period a mass of the order of the entire present ISM will have been lost from the Galaxy. This is a significant but not excessively large mass loss.

Without $C R$ effects taken into account $\left(p_{c o}=0\right)$ one needs for a wind gas temperatures $T_{g o}>8 \cdot 10^{6} \mathrm{kpc}$ at $R_{0}=1 \mathrm{kpc}$, and $T_{g o}>4 \cdot 10^{6} \mathrm{kpc}$ at $R_{0}=10 \mathrm{kpc}$, including centrifugal effects $[16,17,18]$. The average hot ISM is certainly much below such temperatures. However in "supershells" temperatures of such magnitudes might exist [19]. For such regions a purely thermally driven wind might result, as generally for galaxies with sufficiently large hot ISM temperatures. 


\section{Conclusions}

The existence of galactic winds clearly depends on the assumption of a sufficiently small intergalactic pressure. Thus in galaxy clusters with a high intracluster pressure the individual galaxies may not exhibit a wind, whereas the cluster as a whole should show a "cluster wind".

Winds do not constitute a contradiction to fountains on closed or even open field lines ("coronal arcs") in galaxies, or to cooling flows in clusters.

Observations have as yet not given $X$-ray halos indicative of a wind $[20,21]$. On the other hand we interprete the radio halos and the polarization characteristics of NGC 4631 [22], or NGC 891 (Hummel, private communication) as indicative of open magnetic field configurations. These are most likely produced by mass outflows.

\section{References}

1. F. Ipavich: Astrophys. J. 196, 107, (1975)

2. G. Münch, H. Zirin: Astrophys. J. 133, 11 (1961)

3. P.R. Shapiro, G.B. Field: Astrophys. J. 205, 762 (1976)

4. F.D. Kahn: In Investigating the Universe (D. Reidel Publ. Comp.) p. 1 (1981)

5. J.H. Oort: Bull. Astron. Inst. Netherlands 18, 421 (1966)

6. I.F. Mirabel, R. Morras: Astrophys. J. 279, 86 (1984)

7. H. van Woerden, U.J. Schwarz, A.N.M. Hulsbach: In The Milky Way Galaxy, ed. by H. van Woerden et al. (D. Reidel Publ. Comp.), p. 387 (1985)

8. V.L. Ginzburg: Proc. 20th Int. Cosmic Ray Conf. 7,7 (1987)

9. E.N. Parker: Astrophys. J. 145, 811 (1966)

10. J. Skilling: Mon. Not. Roy. Astron. Soc. 172, 557 (1975)

11. H.J. Völk: In High Energy Astrophys., Proc. 19th Rencontre de Moriond, ed. by Tran Than Van (Editions Frontières, Gif-sur-Yvette), p. 281 (1984)

12. J.F. McKenzie, H.J. Völk: Astron. Astrophys. 116, 191 (1982)

13. D. Breitschwerdt, J.F. McKenzie, H.J. Völk: In Interstellar Magnetic Fields, ed. by R. Beck and R. Gräve (Springer-Verlag Berlin, Heidelberg), p. 131 (1987a)

14. D. Breitschwerdt, J.F. McKenzie, H.J. Völk: Proc. 20th Int. Cosmic Ray Conf. $\underline{2}, 115(1987 \mathrm{~b})$

15. J.F. McKenzie, D. Breitschwerdt, H.J. Völk: Proc. 20th Int. Cosmic Ray Conf. $\underline{2}, 119$ (1987)

16. A. Habe, S. Ikeuchi: Progress in Theor. Phys. $\underline{64}, 1995$ (1980)

17. R.A. Chevalier, W.R. Oegerle: Astrophys. J. 227, 398 (1979)

18. J.N. Bregmann: Astrophys. J. 236, 577 (1980)

19. C. Heiles: this volume (1989)

20. D.P. Cox, D. McCammon: Astrophys. J. 304, 657 (1986)

21. J.N. Bregmann, A.E. Glassgold: Astrophys. J. 263, 564 (1982)

22. E. Hummel, H. Lesch, R. Wielebinski, R. Schlickeiser: Astron. Astrophys. 197, L29 (1988) 


\section{Discussion:}

MEBOLD: I want to add the galaxy NGC 1808 to the list of galaxies you used as observational illustrations. Its morphology is suggestive of a poloidad magnetic field symmetrical to the center of the galaxy. Recent observations with the VLA have supported this suggestion.

VÖLK: I have not seen the NGC 1808 morphology up to now and would like to see it. A poloidal field is of course not necessarily open and thus per se does not allow a mass loss to infinity. For that it must be pushed open by an internal "gas" overpressure.

PECKER: If Heiles (and Terlevich) are right, the SN explosions are, and have been, constantly heating local holes in the galactic disk matter; therefore, UV radiation might flow more easily in the halo, and radiation pressure alone (Ly $\alpha$ ) acting on neutral hydrogen might be an efficient "motor" for a wind. How could it compare with your model of galactic C.R. driven galactic wind?

VÖLK: I certanly believe that UV photons will escape into the halo and add to the gas scale height there as well as to the ionization level. I have not compared photon radiation pressure to CR pressure effects but I agree that it should be done. 